\section{Aquaculture farms can have many, highly-variable impacts on nearby wildlife}

Aquaculture farms have the potential to impact nearby wildlife in a number of ways: an individual study alone would be unable to measure all the impacts. So the authors performed a meta-analysis and review of the scientific literature, examining over 9000 research papers published through November 2017, to determine the consensus on both positive and negative impacts from aquaculture farms.

The initial ISI Web of Science and Google Scholar searches for papers were whittled down to 204 studies. To be included in this analysis, studies needed to provide empirical field data on categories of interest (i.e. direct interactions between wildlife and aquaculture stock) so the interactions could be statistically measured. The resulting literature was heavily skewed toward Norway, the US, and Spain, with mainland China notably absent from the selected literature despite the country's enormous aquaculture operations. Marine and estuarine environments were also overrepresented in the literature, despite most aquaculture occurring in freshwater. Fish, especially salmonids, sea bream, and sea bass, were the most studied in the literature. Algal and crustacean aquaculture were barely studied, with only 8 studies between them despite their dominance in the field.

Aquaculture farms' effects on nearby wildlife abundance was varied. Fish were shown to have the largest changes in abundance due to the presence of farms with an average effect size of 49-times what would be expected without the farm. This large effect size was driven by huge effect sizes from a small number of studies (one finding a 1327-times increase) so these effects may be highly variable. The authors found in their meta-analysis that the presence of wildlife near farms was higher for fish farms than shellfish farms. They also found that effect sizes were generally larger if studies compared fish aggregations around farms to featureless reference sites (like the open ocean) versus structured habitats (like rocky reefs).

Farms almost certainly attract fish, either by encouraging wild fish to take up residence near the farms or by encouraging regular visitation. Wild fish found near aquaculture farms were generally 1.2-times larger and 1.7-times heavier than fish found in reference sites. These larger, wild fish were found to have different diets than reference fish, indicating that wild fish living nearby aquaculture operations were likely eating leftover fish food from the cultured fish, or otherwise benefiting from the food inputs to the farm (e.g. through fecal matter).

Three studies investigated antimicrobial-chemical contamination of fish farms, and each of them found such chemicals in the majority of fish sampled. "In each case, mean concentrations for positive samples exceed the current European Union limits for these substances in skin and muscle of finfish for human consumption".

Viral, bacterial, and parasitic infections of wild fish around fish farms was either neutral or elevated. "Of the 11 studies that quantified changes in infection levels with the presence of active fish farms, all found higher levels of infection in farm-associated wild fish, with a mean 16x increase overall".

Aquaculture sites tended to attract birds, particularly cormorants and herons. Shellfish farms tended to attract birds which specialize on eating shellfish or generalists. However,
This is a summary of: Impacts of marine and freshwater aquaculture on wildlife: a global metaanalysis

Accessible at: https://marxiv.org/bpd3a

Authors: Luke Barrett, Stephen Swearer, Tim Dempster

Added to MarXiv: September 2018

Published: Reviews in Aquaculture, 2018

Suggested Citation: Aquaculture farms can have many, highly-variable impacts on nearby wildlife. OCTO (2018). DOI: 10.31230/osf.io/j4ea3

See more MarXiv summaries at https://www.marxivinfo.org/ summaries

Share your research in MarXiv and the MarXiv Team may summarize it, just like this!

Step-by-step instructions on how to share your research in MarXiv are detailed at https://www.marxivinfo.org/ submission

Across all disciplines, papers available for free with a preprint (also known as "Green OA") are cited $30 \%$ more than the world average (DOI: 10.7287/peerj. preprints.3119v1) 


\section{MarXiv Summary}

MarXiv: The free research repository for the ocean \& marine-climate sciences

shellfish farms were noted to possibly displace other types of birds. Predatory birds tended to be killed by entangling themselves in nets or interacting with other anti-predation measures.

Mammals were also noted to be attracted to aquaculture farms: "pinnipeds have a propensity to become 'nuisance animals', damaging nets (leading to fish escapes) and consuming or stressing stock". Steel mesh is the only option to keep mammals out of some farms.

\section{MarXiv}

The MarXiv Team

Nick Wehner,

Director of Open Initiatives,

OCTO

Raye Evrard,

Project Manager, Осто

Allie Brown,

Communications and

Outreach Director, OCTO

Sarah Carr,

Chief Knowledge Broker,

OCTO

John Davis,

President, ОСТО

Correspondence

Nick Wehner

nick@octogroup.org

Financial support for MarXiv comes from the David and Lucile Packard Foundation.

The views expressed herein are those of the MarXiv Team and should not be interpreted as representing the opinions or policies of the Packard Foundation or other funders of MarXiv.

Join the MarXiv Summaries monthly newsletter at https:// oct.to/marxivsum

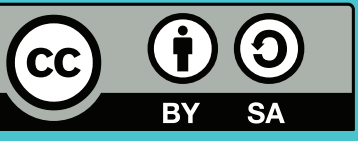

MarXiv is an ОСТO Initiative
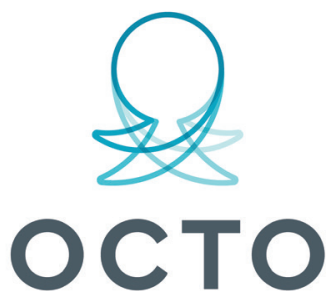

OPEN COMMUNICATIONS FOR THE OCEAN 